\title{
Critical Properties of the Two-Dimensional Villain Model with Ferromagnetic Impurities
}

\author{
Md. Yeakub Ali \\ Department of Mathematics, Chittagong University of Engineering \& Technology, Chittagong, Bangladesh \\ Email: ali69cuet@gmail.com
}

How to cite this paper: Ali, Md.Y. (2017) Critical Properties of the Two-Dimensional Villain Model with Ferromagnetic Impurities. World Journal of Mechanics, 7, 167175 .

https://doi.org/10.4236/wjm.2017.76015

Received: April 24, 2017

Accepted: May 30, 2017

Published: June 2, 2017

Copyright (c) 2017 by author and Scientific Research Publishing Inc. This work is licensed under the Creative Commons Attribution International License (CC BY 4.0).

http://creativecommons.org/licenses/by/4.0/

\begin{abstract}
This work investigates the square lattice in the presence of anti-ferromagnetic exchange considering the two-dimensional Ising Villain model. A computational scheme is constructed to evaluate the degeneracy of ground states at zero temperature employing the Pfaffian method through perturbation theory. The entropy and canonical spin glass of the considered model are precisely obtained. The critical point between the Villain phase and canonical spin glass phase with the line fit of entropy at the thermodynamic limit is investigated. It is also shown that the transition point of the Villain model lies in the low concentration regime.
\end{abstract}

\section{Keywords}

Villain, Entropy, Critical Point, Square Lattice

\section{Introduction}

It is well established that the two-dimensional Ising model is one of the leading non-trivial models, which divulge an exact solution and is widely used for describing physical phenomena in statistical physics. The problems of disordered systems can be studied through the early formulation considered, particularly the perfect Ising lattice by employing the technique. On the other hand, the frustration plays a vital role as a key element to study the disordered system, the socalled spin-glass problem [1]. It may arise from the geometry of the lattice or from a quenched random distribution of positive (ferromagnetic) and negative (anti-ferromagnetic) bonds. For instance, one of the canonical models of frustrated systems is the two-dimensional Ising model. A large number of authors [2]-[7] have studied the canonical model with $p=0.5$ on a square lattice considering various assumption. The spin glass behavior occurs at zero temperature and continues to exist at a critical probability $p_{c}=0.11$ as investigated in 
[8]-[17]. However, a few investigations have developed by extending the method to calculate the spin correlation at zero temperature directly, and the results have obtained by extrapolating the zero-temperature results. Moreover, one may be calculated the ground state free energy and entropy for very large lattice by applying the [13] [18] [19] [20] Pfaffian matrix method, which appears to capture the essence of the physics of the $\pm J$ system. Ali and Poulter [21] have studied the spin-correlations functions of the fully frustrated Ising model and the $\pm J$ Ising spin glass on a square lattice. Blackman and Poulter [19] have investigated that the phase transition of the fully frustrated system is at zero temperature.

Being motivated above the discussion, the purpose of this paper is to present some computational results for the critical behavior of the Villain model [22] brought about in a two-dimensional fully frustrated Ising system by introducing ferromagnetic defects. The antiferromagnetic bonds are replaced instead of ferromagnetic bonds in each unit cell randomly. Some of the results are obtained in different form as the antiferromagnetic bonds increase in the considered model, which are relevant to a particular line of research on spin glass. Spin glass is also characterized by frustration and randomness. There have been a number of studies that have sought to introduce randomness into an initially non-random frustrated system by means of defects. The starting model is fully frustrated with a highly degenerate ground state and the replacement of magnetic sites with a non-magnetic species reduces frustration but inserts randomness. There is a transition from the Villain model to spin glass phase, which is characterized by finite size effect of entropy. Therefore, the paper is organized as follows: model equations and formalism to study the thermodynamic quantity entropy are given in Section 2. The result of this work that comes from extrapolating of entropy is discussed in Section 3. Finally, the conclusion is drawn in Section 4.

\section{Model Equations and Formation}

To study the critical behavior of the Villain model, the two dimensional fully frustrated Villain model [22] can be written in the Hamiltonian form as

$$
H=\sum_{\langle i j\rangle} J_{i j} m_{i} m_{j},
$$

where $J_{i j}$ is the nearest-neighbor interaction of spins and $m_{i}= \pm 1$ is a plaquette $L \times L$ lattice with $N=L^{2}$ spins. The product of the bonds around each plaquette of the system is negative, that is $\prod J_{i j}=-1$. There are also many different ways to do this process which most of them gauge invariant. The exchange of interactions $J_{i j}$ are uncorrelated quenched random variables taking into account the values of $\pm J$. The lattice $L \times L$ is cylindrically wounded on the periodic boundary condition. The model is fully frustrated initially and its ground states are highly degenerates. Furthermore, the ferromagnetic and antiferromagnetic species are completely interacted in much visible system. Moreover, the anti-ferromagnetic interaction may be produced the frustration in the given system. In such system, the replacement of ferromagnetic bond with anti-ferromagnetic bond can be reduced the frustration. The frustration of the sys- 
tem can also lead to the spin glass phase at zero temperature [18] [23] [24] [25] [26] due to defect bonds. Therefore, the probability of nearest neighbor bond distribution [1] [13] [21] [27] [28] of the system may be written as

$$
P\left(J_{i j}\right)=p \delta\left(J_{i j}+J\right)+(1-p) \delta\left(J_{i j}-J\right)
$$

where $p$ is the defect concentration of bond and $\delta=1-\tan h J / T$.

On the other hand, the partition function for the nearest neighbor Ising two dimensional Villain model can be expressed as

$$
Z=2 M_{0} \exp \left(-\frac{E_{0}}{T}\right)\left\{1+\frac{M_{1}}{M_{0}} \exp \left(-\frac{4 J}{T}\right)+\cdots\right\}
$$

where $M_{i}, i=1,2,3, \cdots$ are the degeneracies of the excited states, $E_{0}$ is the ground sate energy and $M_{0}$ is the ground state degeneracy, respectively. The entropy can be evaluated for the given system considering the following formulae as

$$
S=\operatorname{In}\left(2 M_{0}\right)+\frac{M_{1}}{M_{0}} \exp \left(-\frac{4 J}{T}\right)+\frac{4 J M_{1}}{T M_{0}} \exp \left(-\frac{4 J}{T}\right)+\cdots
$$

Finally, the entropy [19] may be extrapolated to an infinite lattice for a lattice of size $L \times L$ and may be defined as

$$
S(L)=S+\beta L^{-1},
$$

where $S(L)$ is the value of the $L \times L$ lattice, $S$ is the entropy in the limit $L \rightarrow \infty$ and $\beta$ is the coefficients of extrapolated entropy.

\section{Results and Discussion}

The results are investigated from the two-dimensional Villain model by replacing the anti-ferromagnetic bonds on the square lattice to employ the Pfaffian approach in the perturbation theory. The square lattices of size $L \times L$ are considered with the range of concentration of negative bonds as $p \in(0.0,0.5)$. The ground-state entropy of the Villain model is obtained accurately in the thermodynamic limit. To investigate the critical behavior of the Villain model, the data are collected and averaged over number of random samples are presented graphically with physical interpretation. The error bars are indicated uncertainties in the figures equal to two standard deviations that is a $95.4 \%$ confidence interval. The uncertainties are subjected to provide in curve fitting by $\chi^{2}$ empolying the Levenberg-Marquardt algorithm [29] [30]. The ground-state entropy with periodic boundary conditions are obtained for the mentioned concentration at different sizes $(L=12,16,24,32,48,64,96,128)$ and $10^{4}$ disorder realizations for $L=12-64$ as well as 5000 disorder for $L=96-128$ considering one replacement of ferromagnetic and anti-ferromagnetic bonds creates one disorder.

Figure 1 shows the ground-state entropy of the Villain model for the lattice sizes $L=16-128, \beta=-0.128$ and $p=0.0$. It is seen that the ferromagnetic and anti-ferromagnetic bonds are fully satisfied in each unit cell, but the concentration of anti-ferromagnetic bond is zero percents. The error bar is not 
visible because the aforementioned model is non-random. It is also seen from Figure 1 that the value of entropy is more accurate at $L \rightarrow \infty$, that is $S=0.2913$, whereas the exact value [31] is 0.2915 . Figure 2 shows that the line fit Equation (5) of entropy crosses parallel to the horizontal line at $L \rightarrow \infty$ for $p=0.011$. The ground-state entropy for $p=0.012$, fitting Equation (5) with

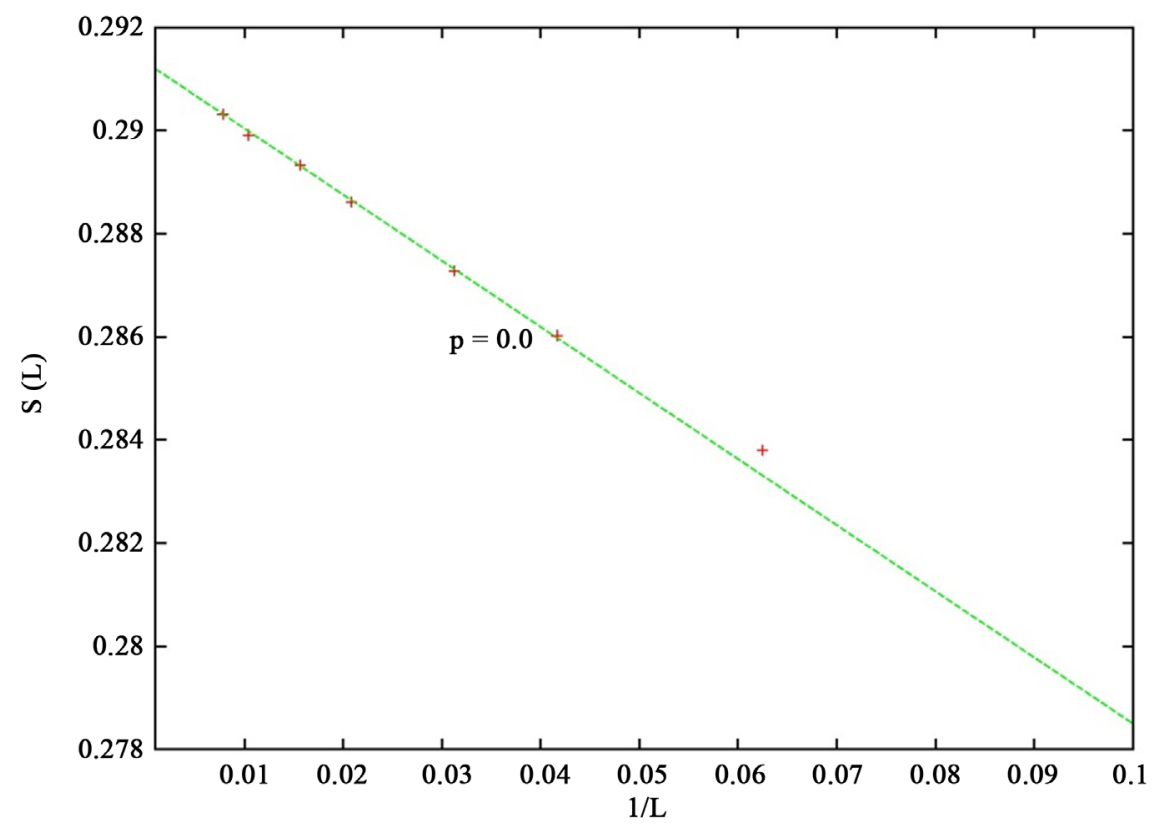

Figure 1. The ground-state entropy for Villain model, fitting Equation (5) with quality of the line fit $Q=1$, gradient $\beta=-0.128$ and different lattice sizes $L$.

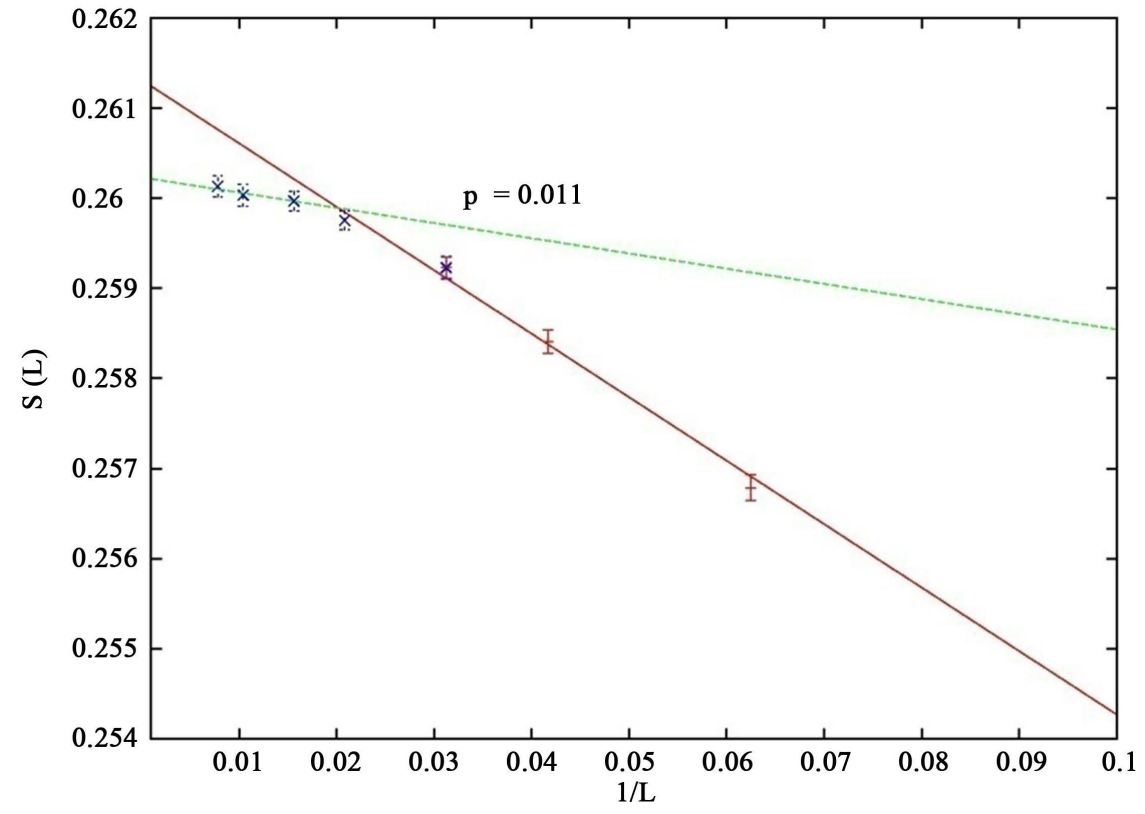

Figure 2. The ground-state entropy for $p=0.011$, fitting Equation (5) with quality of the line fit $Q=0.52$, gradient $\beta=-0.070, L=16-32$ and quality of the line fit $Q=0.92$, gradient $\beta=-0.016, L=48-128$. Here the entropy with error bar for $L=24$ and $L=32$ are almost same. 
quality of the line fit $Q=0.88$, gradient $\beta=-0.069, L=16-32$ and quality of the line fit $Q=0.98$, gradient $\beta=0.0, L=48-128$ is shown in Figure 3 . It is clearly seen from Figure 3 that the entropy turns more flat as $L \rightarrow \infty$. Figure 4 shows the ground-state entropy for $p=0.013$ by fitting Equation (5)

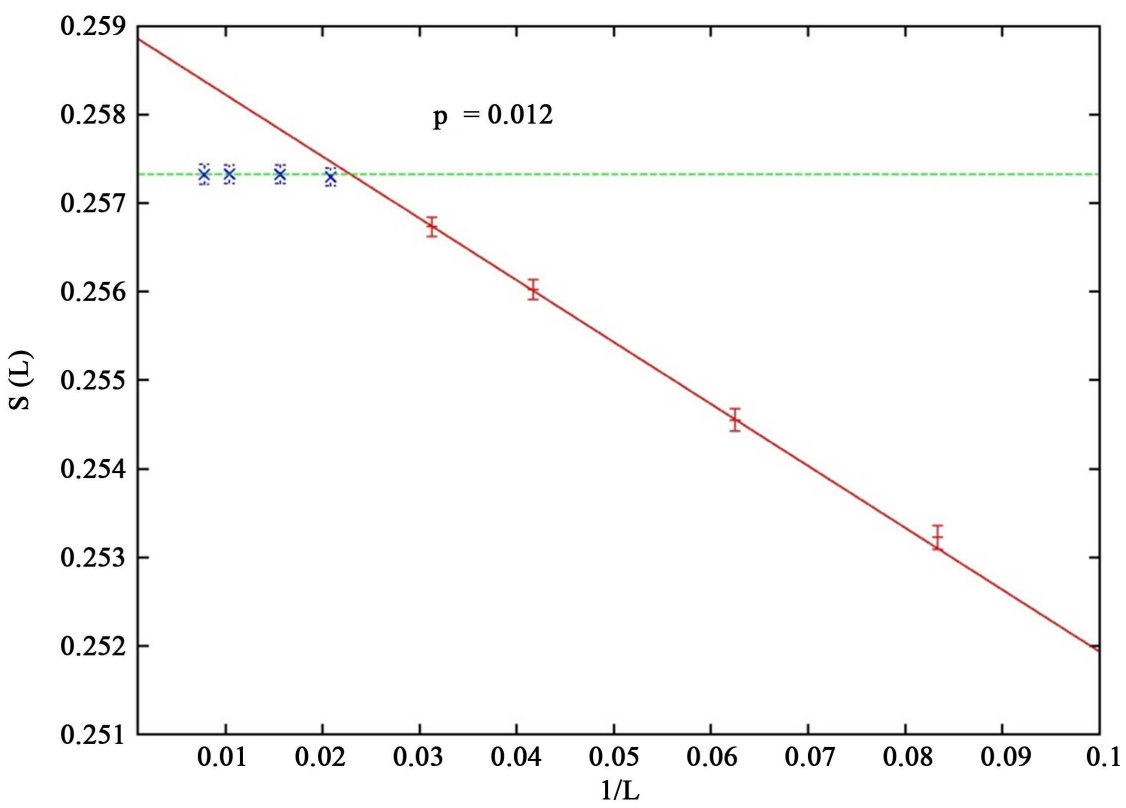

Figure 3. The ground-state entropy for $p=0.012$, fitting Equation (5) with quality of the line fit $Q=0.88$, gradient $\beta=-0.069, L=16-32$ and quality of the line fit $Q=0.98$, gradient $\beta=0.0, L=48-128$.



Figure 4. The ground-state entropy for $p=0.013$, fitting Equation (5) with quality of the line fit $Q=0.62$, gradient $\beta=-0.059, L=16-32$ and quality of the line fit $Q=0.92$, gradient $\beta=0.019, L=48-128$. 
with the quality of the line fit $Q=0.62, \beta=-0.059, L=16-32$ and $Q=0.92, \beta=0.019, L=48-128$. Figure 5 depicts the ground-state entropy for $p=0.014$ with the quality of the line fit $Q=0.75, \beta=-0.053$, $L=16-32$ and $Q=0.96, \beta=0.014, L=48-128$. Moreover, Figure 6 exhibits the ground-state entropy for $p=0.015$ with the quality of the line fit $Q=0.81, \quad \beta=-0.047, L=16-32$ and $Q=0.91, \quad \beta=0.041, L=48-128$. It is observed from Figures 4-6 that the gradient for small $L$ decreases with increasing gradient for larger $L$ at $p=0.013$ to 0.015 . Finally, Figure 7 is showed that the entropy is in good agreement of spin glass for $S=0.0711 \pm 0.0003$ and $p=0.5$ of Ref. [19] in the thermodynamic limit.

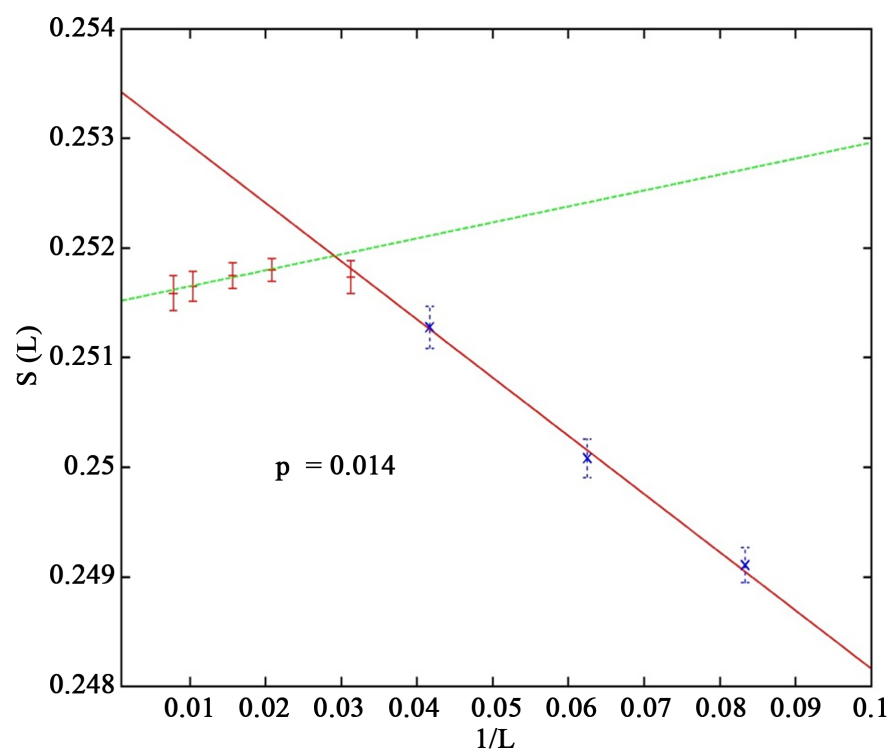

Figure 5. The ground-state entropy for $p=0.014$, fitting Equation (5) with quality of the line fit $Q=0.75$, gradient $\beta=-0.053, L=16-32$ and quality of the line fit $Q=0.96$, gradient $\beta=0.014, L=48-128$.

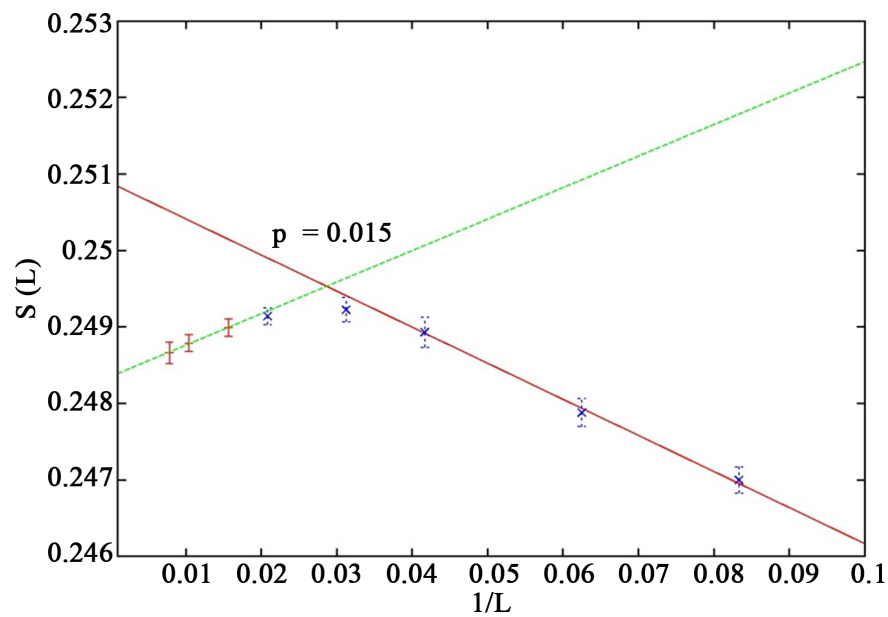

Figure 6. The ground-state entropy for $p=0.015$, fitting Equation (5) with quality of the line fit $Q=0.81$, gradient $\beta=-0.047, L=16-32$ and quality of the line fit $Q=0.91$, gradient $\beta=0.041, L=48-128$. 


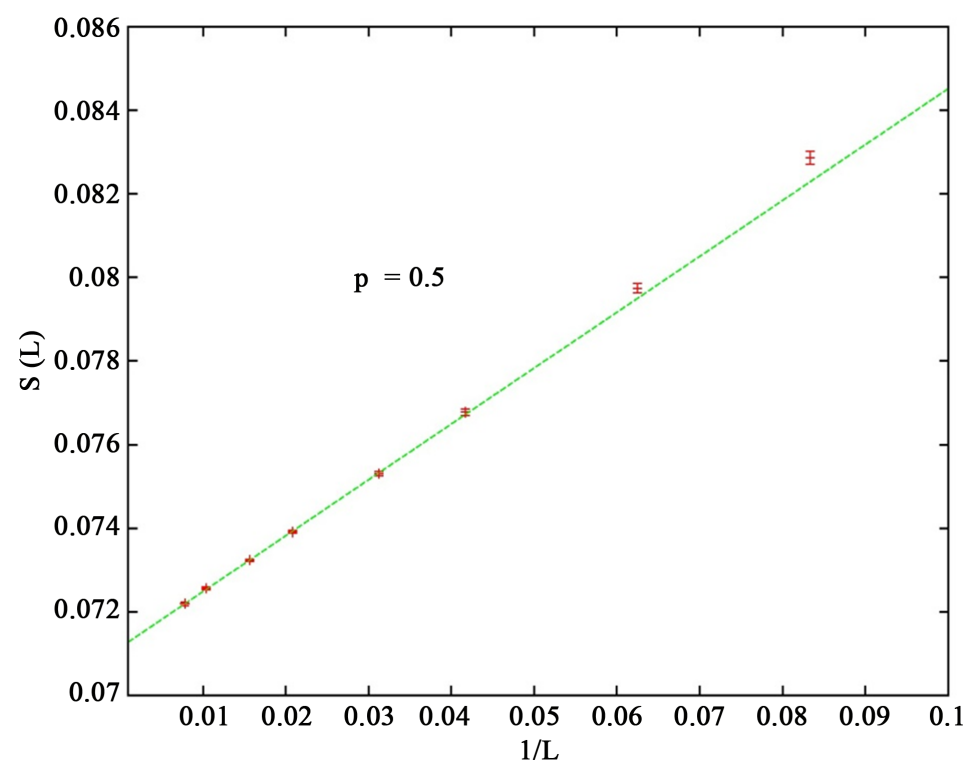

Figure 7. The ground-state entropy for $p=0.5$, fitting Equation (5) with quality of the line fit $Q=0.84$, gradient $\beta=0.133$ and different lattice sizes $L$.

\section{Conclusion}

A numerical computational technique via perturbation method is employed to investigate the critical point of Villain model due to the exchange ferromagnetic defects bond. It is clearly observed that the entropy has behaved different character in the low concentration regime of the Villain model of its line fit at the thermodynamic limit. The two phases, one of which is Villain phase and other one is spin glass phase, are investigated in considered system. The enhanced critical point 0.012 of the two phases is obtained for zero gradients, which is shown in Figure 3. The entropy of the Villain model and canonical spin glass at the thermodynamic limit is also in good agreement with the earlier investigations.

\section{Acknowledgements}

The authors are grateful to the authority of Chittagong University of Engineering \& Technology (CUET). The authors also would like to thanks Professor Dr. Julian Poulter for valuable advice during the research work.

\section{References}

[1] Edwards, S.F. and Anderson, P.W. (1975) Theory of Spin Glasses. Journal of Physics F: Metal Physics, 5, 965. https://doi.org/10.1088/0305-4608/5/5/017

[2] Kawashima, N. and Rieger, H. (1997) Finite-Size Scaling Analysis of Exact Ground States for \pm J Spin Glass Models in Two Dimensions. Europhysics Letters, 39, 85. https://doi.org/10.1209/epl/i1997-00318-5

[3] Kitatani, H. and Sinada, A. (2000) A New Method to Calculate the Spin-Glass Order Parameter of the Two-Dimensional $\pm \mathrm{J}$ Ising Model. Journal of Physics A: Mathematical and General, 33, 3547. https://doi.org/10.1088/0305-4470/33/18/302

[4] Hartmann, A.K. and Young, A.P. (2001) Lower Critical Dimension of Ising Spin 
Glasses. Physical Review B, 64, Article ID: 180404.

https://doi.org/10.1103/PhysRevB.64.180404

[5] Houdayer, J. (2001) A Cluster Monte Carlo Algorithm for 2-Dimensional Spin Glasses. The European Physical Journal B, 22, 479-484.

https://doi.org/10.1007/PL00011151

[6] Sungthong, R. and Poulter, J. (2003) The Critical Temperature of the Two-Dimensional $\pm \mathrm{J}$ Ising Spin Glass. Journal of Physics A: Mathematical and General, 36, 6347. https://doi.org/10.1088/0305-4470/36/24/306

[7] Katzgraber, H.G. and Lee, L.W. (2005) Correlation Length of the Two-Dimensional Ising Spin Glass with Bimodal Interactions. Physical Review B, 71, Article ID: 134404. https://doi.org/10.1103/PhysRevB.71.134404

[8] Morgensterm, I. and Binder, K. (1980) Magnetic Correlations in Two-Dimensional Spin-Glasses. Physical Review B, 22, 288. https://doi.org/10.1103/PhysRevB.22.288

[9] Kirkpatrick, S. (1977) Frustration and Ground-State Degeneracy in Spin Glasses. Physical Review B, 16, 4630. https://doi.org/10.1103/PhysRevB.16.4630

[10] Kitatani, H. and Oguchi, T. (1990) Ferromagnetic-Nonferromagnetic Phase Boundary on the Two-Dimensional $\pm \mathrm{J}$ Ising Model. Journal of the Physical Society of Japan, 59, 3823-3826. https://doi.org/10.1143/JPSJ.59.3823

[11] Ozeki, Y. and Nishimori, H. (1987) Phase Diagram of the \pm J Ising Model in Two Dimensions. Journal of the Physical Society of Japan, 56, 3265-3269. https://doi.org/10.1143/JPSJ.56.3265

[12] Blackman, J.A., Gonçalves, J.R. and Poulter, J. (1998) Properties of the Two-Dimensional Random-Bond $\pm \mathrm{J}$ Ising Spin Glass. Physical Review E, 58, 1502. https://doi.org/10.1103/PhysRevE.58.1502

[13] Ueno, Y. and Ozeki, Y. (1991) Interfacial Approach to d-Dimensional $\pm J$ Ising Models in the Neighborhood of the Ferromagnetic Phase Boundary. Journal of Statistical Physics, 64, 227-249. https://doi.org/10.1007/BF01057875

[14] Aarao Reis, F.D., de Queiroz, S.L.A. and dos Santos, R.R. (1999) Universality, Frustration, and Conformal Invariance in Two-Dimensional Random Ising Magnets. Physical Review B, 60, 6740. https://doi.org/10.1103/PhysRevB.60.6740

[15] Honecker, A., Picco, M. and Pujol, P. (2001) Universality Class of the Nishimori Point in the 2D \pm J Random-Bond Ising Model. Physical Review Letters, 87, Article ID: 047201. https://doi.org/10.1103/PhysRevLett.87.047201

[16] Nobre, F.D. (2001) Phase Diagram of the Two-Dimensional $\pm \mathrm{J}$ Ising Spin Glass. Physical Review E, 64, Article ID: 046108. https://doi.org/10.1103/PhysRevE.64.046108

[17] Merz, F. and Chalker, J.T. (2002) Two-Dimensional Random-Bond Ising Model, Free Fermions, and the Network Model. Physical Review B, 65, Article ID: 054425. https://doi.org/10.1103/PhysRevB.65.054425

[18] Blackman, J.A. (1982) Two-Dimensional Frustrated Ising Network as an Eigenvalue Problem. Physical Review B, 26, 4987. https://doi.org/10.1103/PhysRevB.26.4987

[19] Blackman, J.A. and Poulter, J. (1991) Gauge-Invariant Method for the \pm J Spin-Glass Model. Physical Review B, 44, 4374. https://doi.org/10.1103/PhysRevB.44.4374

[20] Poulter, J. and Blackman, J.A. (2001) Properties of the \pm J Ising Spin Glass on the Triangular Lattice. Journal of Physics A: Mathematical and General, 34, 7527. https://doi.org/10.1088/0305-4470/34/37/308

[21] Ali, M.Y. and Poulter, J. (2013) Spin-Correlation Function of the Fully Frustrated Ising Model and $\pm \mathrm{J}$ Ising Spin Glass on a Square Lattice. Chinese Physics B, 22, Article ID: 067502. https://doi.org/10.1088/1674-1056/22/6/067502 
[22] Villain, J. (1977) Spin Glass with Non-Random Interactions. Journal of Physics C: Solid State Physics, 10, 1717. https://doi.org/10.1088/0022-3719/10/10/014

[23] Atisattapong, W. and Poulter, J. (2008) Energy Gap of the Bimodal Two-Dimensional Ising Spin Glass. New Journal of Physics, 10, Article ID: 093012. https://doi.org/10.1088/1367-2630/10/9/093012

[24] Jinuntuya, N. and Poulter, J. (2012) Elementary Excitations and the Phase Transition in the Bimodal Ising Spin Glass Model. Journal of Statistical Mechanics. Theory and Experiment, 2012, Article ID: P01010. https://doi.org/10.1088/1742-5468/2012/01/P01010

[25] Katzgraber, H.G., Lee, L.W. and Campbell, I.A. (2007) Effective Critical Behavior of the Two-Dimensional Ising Spin Glass with Bimodal Interactions. Physical Review $B$, 75, Article ID: 014412. https://doi.org/10.1103/PhysRevB.75.014412

[26] Kac, M. and Ward, J.C. (1952) A Combinatorial Solution of the Two-Dimensional Ising Model. Physical Review, 88, 1332. https://doi.org/10.1103/PhysRev.88.1332

[27] Goncalves, J.R., Poulter, J. and Blackman, J.A. (1997) Bond and Site Defects in Fully Frustrated Two-Dimensional Ising Systems. Journal of Physics A: Mathematical and General, 30, 2947. https://doi.org/10.1088/0305-4470/30/9/010

[28] Morgenstrem, I. (1982) Phase Transition in the \pm J Ising-Spin-Glass Model. Physical Review B, 25, 6071. https://doi.org/10.1103/PhysRevB.25.6071

[29] Levenberg, K.A. (1944) A Method for the Solution of Certain Non-Linear Problems in Least Squares. Quarterly of Applied Mathematics, 2, 164-168. https://doi.org/10.1090/qam/10666

[30] Marquardt, D. (1964) An Algorithm for Least-Squares Estimation of Nonlinear Parameters. Journal of the Society for Industrial and Applied Mathematics, 11, 431441. https://doi.org/10.1137/0111030

[31] Andre, G., Bidaux, R., Carton, J.-P., Conte, R. and de Seze, L. (1979) Frustration in Periodic Systems: Exact Results for Some 2D Ising Models. Journal de Physique, 40, 479-488. https://doi.org/10.1051/jphys:01979004005047900

Submit or recommend next manuscript to SCIRP and we will provide best service for you:

Accepting pre-submission inquiries through Email, Facebook, LinkedIn, Twitter, etc. A wide selection of journals (inclusive of 9 subjects, more than 200 journals)

Providing 24-hour high-quality service

User-friendly online submission system

Fair and swift peer-review system

Efficient typesetting and proofreading procedure

Display of the result of downloads and visits, as well as the number of cited articles

Maximum dissemination of your research work

Submit your manuscript at: http://papersubmission.scirp.org/

Or contact wjm@scirp.org 